Aus dem Anatomischen Institut der Med. Fakultät, Univ. Okayama (Vorstand: Prof. M. SEKI).

\title{
Die Histometrie des Epithels der Trachea und der Luftwege in der Lunge der Embryonen von Mensch, Kaninchen, Meerschweinchen und Ratte. I.
}

\author{
人，家见，天焦鼠，大熙鼠の氣管と肺內氣道の \\ 上皮の組䄉测定。I.
}

Yoshio KUSUHARA 楠原良雄.

\section{[Eingegangen am 6. Februar 1952.]}

An Untersuchungen über die Histogenese der Lunge sind schon viele vorhanden. Unter anderen sind die Arbeiten von OGAWA (1920), BENDER (1925), POLICARD (1929), SEEMANN (1931) und SHIMAI (1950) zu nennen. Die Trachea und der Bronchus haben aber, weil sie verhältnismäßig einfach strukturiert werden, weniges Interesse geboten. Die Trachea und die Luftwege in der Lunge in der embryonalen Zeit wurden übrigens von Autoren nur stellenweise untersucht, und ein statistisches Studium ist noch nicht unternommen. Die vorliegende Arbeit befaßt sich mit der statistischen Messung der Epithelien der Trachea, des Bronchus und der Bronchulen.

\section{Material und Methode.}

Es standen mir Menschenembryonen von 4, 6 und 8 Monaten, Kaninchenembryonen von 20 und 25 Tagen, ein neugeborenes Kaninchen, ein 3 Wochen altes Kaninchen, Meerschweinchenembryonen von 40 und 50 Tagen, ein neugeborones Meerschweinchen, ein 2 Wochen altes Meerschweinchen, Rattenembryonen von 15 und 18 Tagen, eine neugeborene Ratte und 1 Woche alte Ratte zur Vertügung. Nach der Fixierung in 10\% jger Formalinlösung wurden die Trachea, der Bronchus und die Lunge ausgenommen, in Zelloidin eingebettet, um $15 \mu$ dicke Sohnitte mit Hämatoxylin nnd Eosin zu färben. Die Messung wurde mit dem Okularmikrometer durchgeführt. Es wurde zuerst der Mittelwert der Epitheldicke erzielt. Die Form des Zellkerns läßt manchmal auf die Form des Zellkörpers schließen. Die Zellen mit einem kleinen Größen-Breitenindex (Breite / Länge $\times 100)$ sind nämlich von zylindrischer Form, diejenigen mit einem Wert sehr weit über 100 sind aber platt. Bei den mehrreihigen Epithelien maß man nur die in einer Höhe liegenden Zellkerne. 


\section{Resultate der Messung.}

\section{A. Mensch.}

a) Trachea und Bronchus (Tabelle 1).

Das Epithel ist bei 4-, 6- und 8 monatigen Embryonen immer

Tabelle 1. Epithelien der Trachea und

\begin{tabular}{l|c|c|c|c|c|}
\hline \multirow{2}{*}{} & & \multicolumn{4}{|c}{ Trachea } \\
\cline { 2 - 6 } & $\begin{array}{c}\text { Epithel- } \\
\text { dicke }\end{array}$ & Bau & Kerngröße & Kernbreite \\
\hline \multirow{2}{*}{ 4. fet. M. } & kran. Tl. & 53.4 & \multirow{2}{*}{ mehrreihig } & $6.2 \pm 0.1$ & $4.1 \pm 0.1$ \\
& kaud. Tl. & 39.9 & $6.4 \pm 0.2$ & $4.3 \pm 0.2$ \\
\hline \multirow{2}{*}{ 6. fet. M. } & kran. Tl. & 53.1 & \multirow{2}{*}{ mehrreihig } & $6.6 \pm 0.2$ & $5.5 \pm 0.1$ \\
& kaud. Tl. & 39.9 & & $6.9 \pm 0.1$ & $6.6 \pm 0.2$ \\
\hline \multirow{3}{*}{ 8. fet. M. } & kran. Tl. & 53.1 & & $6.6 \pm 0.1$ & $5.3 \pm 0.1$ \\
& kaud. Tl. & 40.8 & mehrreihig & $6.6 \pm 0.2$ & $5.3 \pm 0.2$ \\
\hline
\end{tabular}

mehrreihig. Aus Tabelle 1 ergibt sich, daß die Epitheldicke der Trachea beim Menschen sich während der Entwicklung, nicht wie bei den anderen Tieren, sich kaum ändert. Die Epitheldicke des Bronchus nimmt im Gegenteil zu den anderen Tieren zu. Der GrößenBreitenindex der im Fpithel hoch stehenden Zellkerne zeigt keine regelmäßige Veränderung.

b) Bronchulen (Tabelle 2).

Die Messung der Bronchulen in der Lunge ist nur in den frühesten Zeiten leicht durchführbar. In späteren Zeiten verästeln sich die Bronchulen wiederholt, und die Endstücke der letzteren erweitern sich beträchtlich, deren Zellen kubisch bis platt werden. Eine statistische Messung wird dann sehr schwer, zumal da die Lungenalveolen, worauf schon vor allem POLICARD (1929) aufmerksam gemacht hat, wieder der klassischen Theorie ursprünglich in dem Bindegewebe entstehen und im postnatalen Leben nur mit diskontinuierlichem Epithel bekleidet sind. Daher befindet sich die Luft in direktem Kontakt mit dem blutgefäßreichen Bindegewebe. In der Tat ist uns eine einwandfreie Darstellung sog. kernloser Platten in der Alveolarwand der Săugetiere durch die Versilberung nicht gelungen. Dazu kommt noch, daß die Zellform und -größe der Bronchulenwandung gan\% mannigfaltig werden, so $\mathrm{d} a \mathrm{~B}$ die Variation der gemessenen Werte stark wächst. Es scheint also unbegründet, den arithmetischen Mittelwert der Einzelwerte der Epithelien der Bronchulen tadellos zu suchen. In 
vorliegender Arbeit wählte man also in den Schnittpräparaten unsachgemäß einige Bronchulen und maß die Epitheldicke und Kerngröße an jedem Bronchulus und ermittelte die Mittelwerte. Die Untersuchungen über die Epithelien der terminalen Bronchulen und der Alveolargängen werden hier ausgerommen.

des Bronchus des Menschen $(\mu)$.

\begin{tabular}{c|c|c|c|c|c}
\hline & \multicolumn{5}{|c}{ Bronchus } \\
\hline $\begin{array}{c}\text { Größen- } \\
\text { Breiten- } \\
\text { index }\end{array}$ & $\begin{array}{c}\text { Epithel- } \\
\text { dicke }\end{array}$ & Bau & Kerngröße & Kernbreite & $\begin{array}{c}\text { Größen- } \\
\text { Breiten- } \\
\text { index }\end{array}$ \\
\hline 66 & 39.0 & mehrreihig & $6.1 \pm 0.2$ & $4.4 \pm 0.2$ & 72 \\
67 & 39.3 & $6.0 \pm 0.2$ & $4.6 \pm 0.2$ & 77 \\
\hline 83 & 47.7 & mehrreihig & $6.2 \pm 0.2$ & $5.8 \pm 0.2$ & 94 \\
96 & 45.9 & & $6.2 \pm 0.2$ & $5.8 \pm 0.2$ & 85 \\
\hline 80 & 50.1 & mehrreihig & $6.3 \pm 0.1$ & $4.8 \pm 0.1$ & 76 \\
82 & 46.8 & & $6.3 \pm 0.2$ & $4.4 \pm 0.2$ & 70
\end{tabular}

Tabelle 2. Epithel der Bronchulen des Menschen $(\mu)$.

\begin{tabular}{l|c|c|c|c|c|c}
\hline & $\begin{array}{c}\text { Durch- } \\
\text { messer }\end{array}$ & $\begin{array}{c}\text { Epithel- } \\
\text { dicke }\end{array}$ & Bau & $\begin{array}{c}\text { Kern- } \\
\text { größe }\end{array}$ & $\begin{array}{c}\text { Kern- } \\
\text { breite }\end{array}$ & $\begin{array}{c}\text { Größen- } \\
\text { Breiten- } \\
\text { index }\end{array}$ \\
\hline \multirow{3}{*}{ 4. fet. M. } & 360 & 25.2 & & 5.4 & 5.4 & 100 \\
& 330 & 24.6 & $\begin{array}{c}\text { meist } \\
2-\end{array}$ & 6.9 & 5.6 & 81 \\
& 132 & 21.0 & reih ig & 6.8 & 6.1 & 90 \\
& 109 & 21.9 & & 6.4 & 5.8 & 91 \\
\hline \multirow{3}{*}{ 6. fet. M. } & 228 & 21.9 & & 7.1 & 6.2 & 87 \\
& 156 & 22.0 & meist & 6.6 & 6.0 & 91 \\
& 135 & 20.1 & reihig & 7.1 & 6.0 & 85 \\
& 132 & 21.8 & & 6.8 & 7.1 & 96 \\
\hline \multirow{3}{*}{ 8. fet. M. } & 333 & 20.1 & & 7.5 & 5.9 & 79 \\
& 264 & 18.7 & meist & 7.1 & 5.2 & 73 \\
& 255 & 19.5 & reihig & 7.4 & 5.5 & 74 \\
& 234 & 18.7 & & 7.3 & 5.7 & 78
\end{tabular}

Was oben bei der Untersuchung der Bronchulen des Menschen gesagt ist, gilt von den Bronchulen anderer Tiere.

In Tabelle 2 ersieht man, daß das von mir untersuchte Bronchulenepithel meist zweireihig bleibt. Der Größen-Breitenindex der im 
Epithel hoch liegenden Kerne nimmt in späteren Embryonalzeitetwas ab.

\section{B. Kaninchen.}

a) Trachea und Bronchus (Tabelle 3).

Tabelle 3. Epithelien der Trachea

\begin{tabular}{|c|c|c|c|c|c|}
\hline & & \multicolumn{4}{|c|}{ Trachea } \\
\hline & & $\begin{array}{l}\text { Epithel- } \\
\text { dicke }\end{array}$ & Bau & $\begin{array}{l}\text { Kern- } \\
\text { größe }\end{array}$ & $\begin{array}{l}\text { Kern- } \\
\text { breite }\end{array}$ \\
\hline \multirow{2}{*}{ 20. fet. $\mathrm{Tg}$. } & kran. Tl. & 19.8 & 2-reihig & $6.9 \pm 0.0$ & $6.0 \pm 0.1$ \\
\hline & kaud. Tl. & 19.2 & $\begin{array}{l}\text { 2-bis } \\
\text { 3-reihig }\end{array}$ & $6.9 \pm 0.1$ & $5.4 \pm 0.1$ \\
\hline \multirow{2}{*}{ 25. fet. $\mathrm{Tg}$. } & kran. Tl. & 19.8 & 2-reihig & $6.9 \pm 0.1$ & $5.2 \pm 0.2$ \\
\hline & kand. Tl. & 19.5 & 2-reihig & $6.5 \pm 0.2$ & $5.3 \pm 0.2$ \\
\hline \multirow{2}{*}{$\begin{array}{c}\text { Neu- } \\
\text { geborenes }\end{array}$} & kran. Tl. & 15.3 & 2-reihig & $6.8 \pm 0.1$ & $5.5 \pm 0.2$ \\
\hline & kaud. Tl. & 15.0 & 2-reihig & $6.5 \pm 0.1$ & $5.4 \pm 0.2$ \\
\hline \multirow{2}{*}{$\begin{array}{c}3 \text { Wochen } \\
\text { n. P. }\end{array}$} & kran. TI. & 14.4 & 2-reihig & $6.9 \pm 0.1$ & $5.7 \pm 0.2$ \\
\hline & kaud. Tl. & 14.1 & 2-reihig & $7.0 \pm 0.1$ & $5.7 \pm 0.2$ \\
\hline
\end{tabular}

Sowohl in der Embryonalzeit als auch nach dem Partus ist das Epithel zweireihig. Fs ist im Bronchus immer dünner als in der Trachea. Dementsprechend erweist sich der Größen-Breitenindex der

Tabelle 4. Epithel der Bronchulen des Kaninchens $(\mu)$.

\begin{tabular}{c|c|c|c|c|c|c}
\hline & $\begin{array}{c}\text { Durch- } \\
\text { messer }\end{array}$ & $\begin{array}{c}\text { Epithel- } \\
\text { dicke }\end{array}$ & Ball & $\begin{array}{c}\text { Kern- } \\
\text { gröBe }\end{array}$ & $\begin{array}{c}\text { Kern- } \\
\text { breite }\end{array}$ & $\begin{array}{c}\text { Größen- } \\
\text { Breiten- } \\
\text { index }\end{array}$ \\
\hline \multirow{5}{*}{ 20. fet. Tg. } & 255 & 13.7 & & 5.6 & 5.6 & 100 \\
& 48 & 11.9 & & 5.8 & 4.4 & 76 \\
& 30 & 13.0 & & 5.0 & 3.9 & 78 \\
& 27 & 11.5 & & 5.5 & 4.4 & 80 \\
& 24 & 11.5 & ein- & 5.6 & 5.0 & 90 \\
& 24 & 11.6 & & 6.0 & 5.3 & 88 \\
& 21 & 10.7 & & 5.6 & 4.3 & 77 \\
& 18 & 11.0 & & 5.9 & 4.6 & 78 \\
\hline
\end{tabular}


hoch liegenden Zellkerne im Bronchus etwas größer als in der Trachea. Der Index wert verändert sich wenig während der Entwick]ung.

b) Bronchulen (Tabelle 4).

Das Epithel ist meist einschichtig. Die Zellkerne zeigen keine regelmäßige Veränderung.

und des Bronchus des Kaninchens $(\mu)$.

\begin{tabular}{|c|c|c|c|c|c|c|c|}
\hline & \multicolumn{7}{|c|}{ Bronchus } \\
\hline $\begin{array}{l}\text { Größen- } \\
\text { Breiten- } \\
\text { index }\end{array}$ & $\begin{array}{l}\text { Epithel- } \\
\text { dicke }\end{array}$ & Bau & \multicolumn{2}{|c|}{ Kerngröße } & \multicolumn{2}{|c|}{ Kernbreite } & $\begin{array}{l}\text { Größen- } \\
\text { Breiten- } \\
\text { index }\end{array}$ \\
\hline 82 & 19.2 & \multirow{2}{*}{$\begin{array}{l}\text { 2-bis } \\
\text { 3-reihig } \\
\text { 2-bis } \\
\text { 3-reihis }\end{array}$} & \multicolumn{2}{|c|}{$6.2 \pm 0.2$} & \multicolumn{2}{|c|}{$5.6 \pm 0.2$} & 90 \\
\hline 81 & 18.3 & & \multicolumn{2}{|c|}{$6.2 \pm 0.2$} & \multicolumn{2}{|c|}{$5 . ? \pm 0.1$} & 86 \\
\hline 75 & 18.6 & 2-reihig & \multicolumn{2}{|c|}{$6.2 \pm 0.2$} & \multicolumn{2}{|c|}{$5.3 \pm 0.1$} & 86 \\
\hline 82 & 18.3 & 2-reihig & \multicolumn{2}{|c|}{$6.2 \pm 0.2$} & \multicolumn{2}{|c|}{$5.3 \pm 0.1$} & 86 \\
\hline 81 & 14.7 & $\begin{array}{l}\text { 2-bis } \\
\text { 3-reihig }\end{array}$ & \multicolumn{2}{|c|}{$6.2 \pm 0.2$} & \multicolumn{2}{|c|}{$5.5 \pm 0.2$} & 89 \\
\hline 83 & 14.1 & 2-reihig & \multicolumn{2}{|c|}{$6.2 \pm 0.1$} & \multicolumn{2}{|c|}{$5.6 \pm 0.2$} & 90 \\
\hline 83 & 14.4 & 2-reihig & \multicolumn{2}{|c|}{$6.0 \pm 0.1$} & \multicolumn{2}{|c|}{$6.0 \pm 0.3$} & 100 \\
\hline 82 & 13.5 & 2-reihig & \multicolumn{2}{|c|}{$6.2 \pm 0.2$} & \multicolumn{2}{|c|}{$6.2 \pm 0.2$} & 100 \\
\hline \multicolumn{8}{|c|}{ Tabelle 4 (Fortsetzung). } \\
\hline \multirow{9}{*}{ 25. tet. $\mathrm{Tg}$. } & 162 & 10.4 & \multirow{9}{*}{$\begin{array}{l}\text { ein- } \\
\text { schicht. }\end{array}$} & 5. & & 4.9 & 91 \\
\hline & 144 & 10.4 & & 5. & & 5.0 & 89 \\
\hline & 114 & 10.1 & & 5 . & & 4.3 & 81 \\
\hline & 99 & 10.3 & & 5. & & 4.5 & 85 \\
\hline & 36 & 11.5 & & 5. & & 4.1 & $8: 2$ \\
\hline & 30 & 11.2 & & 5. & & 4.7 & 89 \\
\hline & 27 & 12.2 & & 5. & & 4.5 & 82 \\
\hline & 24 & 10.6 & & 4. & & 4.0 & 83 \\
\hline & 21 & 10.3 & & 4. & & 3.7 & 76 \\
\hline \multirow{9}{*}{$\begin{array}{l}\text { Neu- } \\
\text { geborenes }\end{array}$} & 243 & 12.3 & & 5. & & 5.1 & 91 \\
\hline & 156 & 13.7 & & 5. & & 4.8 & 86 \\
\hline & 81 & 10.8 & & 5. & & 4.3 & 84 \\
\hline & 42 & 9.8 & & 4. & & 4.1 & 86 \\
\hline & 33 & 9.8 & schicht. & 4. & & 3.7 & 88 \\
\hline & 30 & 10.1 & & 4. & & 4.5 & 92 \\
\hline & 27 & 10.3 & & 4. & & 4.1 & 98 \\
\hline & 24 & 9.9 & & 4. & & 8.8 & 86 \\
\hline & 21 & 10.2 & & 4. & & 4.2 & 88 \\
\hline
\end{tabular}


Tabelle 4 (Fortsetzung).

\begin{tabular}{l|r|r|r|r|r|r}
\hline & 7.3 & 9.3 & & 5.5 & 4.4 & 80 \\
& 69 & 9.6 & & 5.4 & 4.2 & 78 \\
\hline \multirow{3}{*}{ Wochen } & 48 & 9.5 & & 5.4 & 4.4 & 82 \\
n.P. & 47 & 7.8 & ein- & 5.0 & 4.2 & 84 \\
& 36 & 10.3 & schicht. & 5.7 & 5.5 & 97 \\
& 30 & 8.9 & & 5.2 & 4.3 & 83 \\
& 30 & 8.2 & & 5.1 & 4.2 & 82 \\
& 24 & 9.2 & & 4.9 & 4.2 & 86 \\
& & & 5.5 & 4.5 & 82
\end{tabular}

Tabelle 5. Epithelien der Trachea und

\begin{tabular}{|c|c|c|c|c|c|}
\hline & & \multicolumn{4}{|c|}{ Trachea } \\
\hline & & $\begin{array}{l}\text { Epithel- } \\
\text { dicke }\end{array}$ & Bau & Kerngröße & Kernbreite \\
\hline \multirow{2}{*}{ 40. fet. Ty. } & & & $\begin{array}{l}2 \text { - bis } \\
\text { 3-reihig }\end{array}$ & $6.7 \pm 0.2$ & $6.6 \pm 0.1$ \\
\hline & kaud. Tl. & 28.2 & $\begin{array}{l}\text { 2- bis } \\
\text { 3-reihig }\end{array}$ & $6.6 \pm 0.2$ & $6.5 \pm 0.2$ \\
\hline \multirow{2}{*}{ 50. fet. $\mathbf{T g}$} & & & $\begin{array}{c}3-\text { bis } \\
\text { 4-reihig }\end{array}$ & $6.3 \pm 0.2$ & $6.3 \pm 0.2$ \\
\hline & kaud. Tl. & 30.0 & $\begin{array}{l}3-\text { bis } \\
\text { 4-reihiy }\end{array}$ & $6.3 \pm 0.2$ & $6.2 \pm 0.1$ \\
\hline \multirow{2}{*}{$\begin{array}{l}\text { Neu. } \\
\text { geborenes }\end{array}$} & kran. Tl. & 29.1 & $\begin{array}{l}\text { 3-bis } \\
\text { 4-reihig }\end{array}$ & $6.3 \pm 0.2$ & $6.2 \pm 0.2$ \\
\hline & kaud. Tl. & 28.5 & $\begin{array}{l}3 \text { bis } \\
\text { 4-reihig }\end{array}$ & $6.3 \pm 0.2$ & $5.3 \pm 0.2$ \\
\hline \multirow{2}{*}{$\begin{array}{l}2 \text { Wochen } \\
\text { n. P. }\end{array}$} & kran. $\mathrm{Tl}$ & 26.4 & $\begin{array}{l}\text { 3-bis } \\
\text { 4-reihig }\end{array}$ & $6.4 \pm 0.2$ & $5.4 \pm 0.2$ \\
\hline & kaud. Tl. & 25.8 & $\begin{array}{l}\text { 3- bis } \\
\text { 4-reihig }\end{array}$ & $6.4 \pm 0.2$ & $6.4 \pm 0.2$ \\
\hline
\end{tabular}

\section{Meerschweinchen.}

a) Trachea und Bronchus (Tabelle 5).

Tabelle 6. Epithel der Bronchulen des Meerschweinchens ( $\mu$ ).

\begin{tabular}{|c|c|c|c|c|c|c|}
\hline & $\begin{array}{l}\text { Durch- } \\
\text { messer }\end{array}$ & $\begin{array}{l}\text { Epithel- } \\
\text { dicke }\end{array}$ & Bau & $\begin{array}{l}\text { Kern- } \\
\text { größe }\end{array}$ & $\begin{array}{l}\text { Kern- } \\
\text { breite }\end{array}$ & $\begin{array}{l}\text { Größen- } \\
\text { Breiten- } \\
\text { index }\end{array}$ \\
\hline \multirow{7}{*}{ 40. fet. Tg. } & 75 & 13.0 & \multirow{5}{*}{$\begin{array}{c}\text { meist } \\
\text { ein- } \\
\text { schicht. }\end{array}$} & 6.2 & 5.1 & 82 \\
\hline & 45 & 12.8 & & 5.8 & 5.4 & 93 \\
\hline & 27 & 11.9 & & 6.1 & 5.5 & 90 \\
\hline & 24 & 12.1 & & 5.9 & 5.7 & 97 \\
\hline & 21 & 11.0 & & 6.2 & 5.4 & 87 \\
\hline & 69 & 13.6 & & 6.1 & 5.3 & S:2 \\
\hline & 36 & 11.5 & & 5.8 & 5.1 & 83 \\
\hline
\end{tabular}


Hier ist das Epithel wie bei dem Kaninchen vor und nach dem Partus immer 2-3 reihig. Es neigt dazu, nach dem Partus dünner zu werden. Der Größen-Breitenindex der hoch liegenden Epithelkerne ist im Bronchus immer kleiner als in der Trachea.

b) Bronchulen ('Tabelle 6 ).

Das untersuchte Epithel besteht immer aus einschichtigen Zylinderzellen und wird etwas dünner mit dem Schreiten der Entwicklung. Die Zellkerne zeigen keine regelmäßige Veränderung des GrößenBreitenindexes.

des Bronchus des Meerschweinchens $(\mu)$.

\begin{tabular}{|c|c|c|c|c|c|}
\hline \multirow[b]{2}{*}{$\begin{array}{l}\text { Größen- } \\
\text { Breiten- } \\
\text { index }\end{array}$} & \multicolumn{5}{|c|}{ Bronchus } \\
\hline & $\begin{array}{l}\text { Epithel. } \\
\text { dicke }\end{array}$ & Bau & Kerngröße & Kernbreite & $\begin{array}{l}\text { (irößen- } \\
\text { Breiten- } \\
\text { index }\end{array}$ \\
\hline 98 & 27.1 & $\begin{array}{l}2 \text { 2-bis } \\
\text { 3-reihig }\end{array}$ & $6.5 \pm 0.1$ & $5.5 \pm 0.2$ & 85 \\
\hline 99 & 26.1 & $\begin{array}{l}\text { 2-bis } \\
\text { 3-reihig }\end{array}$ & $6.5 \pm 0.1$ & $5.4 \pm 0.2$ & 83 \\
\hline 100 & 28.8 & $\begin{array}{l}\text { 3- bis } \\
\text { 4-reihig }\end{array}$ & $6.4 \pm 0.2$ & $5.3 \pm 0.2$ & 83 \\
\hline 99 & 29.4 & $\begin{array}{l}\text { 3-bis } \\
\text { 4-reihig }\end{array}$ & $6.5 \pm 0.2$ & $5.4 \pm 0.1$ & 83 \\
\hline 99 & 27.0 & $\begin{array}{l}\text { 3- bis } \\
\text { 4-reihig }\end{array}$ & $6.5 \pm 0.2$ & $5.2 \pm 0.2$ & 80 \\
\hline 84 & 26.4 & $\begin{array}{l}\text { 3-bis } \\
\text { 4-reihig }\end{array}$ & $6.5 \pm 0.1$ & $5.4 \pm 0.2$ & 83 \\
\hline 84 & 25.2 & $\begin{array}{l}\text { 3-bis } \\
\text { 4-reihig }\end{array}$ & $6.3 \pm 0.2$ & $5.2 \pm 0.1$ & 83 \\
\hline 100 & 24.9 & $\begin{array}{l}\text { 3-bis } \\
\text { 4-reihig }\end{array}$ & $6.3 \pm 0.2$ & $5.2 \pm 0.2$ & 83 \\
\hline
\end{tabular}

Tabelle 6 (Fortsetzung).

\begin{tabular}{c|c|c|c|c|c|c} 
50. fet. Tg. & 33 & 15.6 & $\begin{array}{c}\text { meist } \\
\text { ein- }\end{array}$ & 4.8 & 2.6 & 78 \\
& 30 & 10.9 & schicht. & 5.8 & 5.2 & 90 \\
& 21 & 13.7 & & 6.2 & 4.9 & 79 \\
\hline & 36 & 12.3 & & 5.7 & 5.0 & 88 \\
& 35 & 12.7 & meist & 6.2 & 5.3 & 86 \\
$\begin{array}{c}\text { Neu- } \\
\text { geborenes }\end{array}$ & 33 & 11.8 & $\begin{array}{c}\text { ein. } \\
\text { schicht. }\end{array}$ & 5.1 & 4.2 & 82 \\
& 30 & 11.4 & & 5.6 & 4.5 & 80 \\
& 21 & 13.5 & & 5.4 & 4.4 & 82 \\
\hline & 162 & 10.9 & & 5.5 & 4.7 & 86 \\
2 Wochen & 63 & 11.0 & meist & 5.0 & 4.9 & 98 \\
n.P. & 48 & 13.5 & ein- & 5.6 & 4.2 & 75 \\
& 45 & 12.4 & schicht. & 5.3 & 5.2 & 98 \\
& 15 & 11.9 & & 5.7 & 5.3 & 93
\end{tabular}




\section{I). Ratte.}

a) Trachea und Bronchus (Tabelle 7).

Die Epithelzellen ordnen sich in der Embryonalzeit 2-3 reihig und nach dem Partus $1-2$ reihig. Das Epithel des Bronchus ist etwas

Tabelle 7. Epithelien der Trachea

\begin{tabular}{|c|c|c|c|c|c|}
\hline & & \multicolumn{4}{|c|}{ Trachea } \\
\hline & & $\begin{array}{l}\text { Epithel- } \\
\text { dicke }\end{array}$ & Bau & Kerngröße & Kernbreite \\
\hline \multirow{2}{*}{ 15. fet. $\mathrm{Tg}$. } & $\mathrm{kran} . \mathbf{T}$ & 20.4 & $\begin{array}{l}\text { 2- bis } \\
\text { 3-reihig }\end{array}$ & $6.8 \pm 0.2$ & $5.6 \pm 0.2$ \\
\hline & kaud. Tl. & 17.7 & $\begin{array}{l}\text { 2-bis } \\
\text { s-reihig }\end{array}$ & $6.9 \pm 0.3$ & $5.7 \pm 0.2$ \\
\hline \multirow{2}{*}{ 18. fet. $\mathrm{Tg}$. } & kran. Tl. & 17.1 & $\begin{array}{c}2 . \text { bis } \\
\text { 3-reihng }\end{array}$ & $6.3 \pm 0.1$ & $5.7+0.2$ \\
\hline & kaud. Tl. & 16.2 & $\begin{array}{l}\text { 2- bis } \\
\text { 3-reihig }\end{array}$ & $6.9 \pm 0.1$ & $5.7 \pm 0.2$ \\
\hline \multirow{2}{*}{$\begin{array}{l}\text { Neu. } \\
\text { geborenes }\end{array}$} & kran. Tl. & 16.2 & $\begin{array}{c}\text { 1-bis } \\
\text { 2-reihig }\end{array}$ & $6.6 \pm 0.1$ & $5.9 \pm 0.2$ \\
\hline & kaud. Tl. & 12.6 & $\begin{array}{l}\text { 1-bis } \\
\text { 2-reihig }\end{array}$ & $6.5 \pm 0.2$ & $6.3 \pm 0.2$ \\
\hline \multirow{2}{*}{$\begin{array}{l}1 \text { Woche } \\
\text { n. P. }\end{array}$} & kran. Tl. & 12.3 & $\begin{array}{c}\text { 1-bis } \\
\text { 2-reiligg }\end{array}$ & $6.8 \pm 0.2$ & $6.1 \pm 0.2$ \\
\hline & kaud. Tl. & 9.9 & $\begin{array}{c}\text { 1-bis } \\
\text { 2-reihig }\end{array}$ & $6.6 \pm 0.2$ & $5.2 \pm 0.2$ \\
\hline
\end{tabular}

dünner als das der Trachea. Die beiden Epithelien werden mit dem Zeilablauf dünner. Der Größen-Breitenindex der Zellkerne bleibt immer etwa gleich.

Tabelle 8. Epithel der Bronchulen der

Ratte $(\mu)$.

\begin{tabular}{c|c|c|c|c|c|c}
\hline & $\begin{array}{c}\text { Durch- } \\
\text { messer }\end{array}$ & $\begin{array}{c}\text { Epithel- } \\
\text { dicke }\end{array}$ & Bau & $\begin{array}{c}\text { Kern- } \\
\text { größe }\end{array}$ & $\begin{array}{c}\text { Kern- } \\
\text { breite }\end{array}$ & $\begin{array}{c}\text { Größen- } \\
\text { Breiten- } \\
\text { index }\end{array}$ \\
\hline \multirow{3}{*}{ 15. fet. Tg. } & 25 & 14.4 & & 6.0 & 4.7 & 78 \\
& 29 & 13.3 & & 5.7 & 5.1 & 90 \\
& 28 & 13.8 & & 6.0 & 4.8 & 80 \\
& 26 & 14.1 & & 6.1 & 5.1 & 84 \\
& 25 & 14.0 & ein- & 5.7 & 4.8 & 84 \\
& 20 & 14.6 & schicht. & 6.0 & 5.2 & 87 \\
& 20 & 15.0 & & 5.8 & 4.5 & 78 \\
& 19 & 13.7 & & 5.9 & 4.8 & 8.2 \\
& 19 & 13.5 & & 1.0 & 5.1 & 85 \\
& 12 & 11.9 & & 5.6 & 5.0 & 89 \\
\hline
\end{tabular}


b) Bronchulen (Tabelle 8).

Die Bronchulen sind mit einschichtigen Zylinderepithelzellen ausgekleidet. Der Größen-Breitenindex ist in der prä- und postnatalen Zeit fast der gleiche.

und des Bronchus der Ratte $(\mu)$.

\begin{tabular}{|c|c|c|c|c|c|}
\hline & \multicolumn{5}{|c|}{ Bronchus } \\
\hline $\begin{array}{l}\text { Grüßen- } \\
\text { Breiten- } \\
\text { index }\end{array}$ & $\begin{array}{l}\text { Epithel- } \\
\text { dicke }\end{array}$ & Bau & Kerngröße & Kernbreite & $\begin{array}{l}\text { Größen- } \\
\text { Breiten- } \\
\text { index }\end{array}$ \\
\hline 82 & 17.1 & $\begin{array}{l}\text { 2-bis } \\
\text { 3-reihig }\end{array}$ & $6.4 \pm 0.1$ & $5.6 \pm 0.2$ & 88 \\
\hline 83 & 16.2 & $\begin{array}{l}\text { 2-bis } \\
\text { 3-reihig }\end{array}$ & $6.5 \pm 0.1$ & $5.7 \pm 0.2$ & 88 \\
\hline 91 & 15.3 & $\begin{array}{l}\text { 2-bis } \\
\text { 3-relhig }\end{array}$ & $6.8 \pm 0.2$ & $5.7 \pm 0.2$ & 84 \\
\hline 83 & 15.0 & $\begin{array}{c}\text { 2-bis } \\
\text { 3-reihig }\end{array}$ & $6.2 \pm 0.3$ & $5.7 \pm 0.2$ & 92 \\
\hline 89 & 12.3 & $\begin{array}{c}\text { 1-bis } \\
\text { 2-reihig }\end{array}$ & $6.7 \pm 0.1$ & $5.3 \pm 0.2$ & 79 \\
\hline 97 & 11.4 & $\begin{array}{l}1 \text { - bis } \\
2 \text {-reihig }\end{array}$ & $5.5 \pm 0.2$ & $6.3 \pm 0.3$ & $9 \cdot 3$ \\
\hline 90 & 9.9 . & $\begin{array}{l}\text { 1. bis } \\
\text { 2-reihig }\end{array}$ & $6.4 \pm 0.2$ & $5.9 \pm 0.2$ & 92 \\
\hline 79 & 9.0 & $\begin{array}{c}\text { 1-bis } \\
\text { 2-reihig }\end{array}$ & $5.4 \pm 0.2$ & $5.4 \pm 0.2$ & 100 \\
\hline
\end{tabular}

Tabelle 8 (Fotrsetzung).

\begin{tabular}{|c|c|c|c|c|c|c|}
\hline \multirow{10}{*}{ 18. fet. $\mathrm{Tg}$. } & 33 & 13.2 & \multirow{10}{*}{$\begin{array}{l}\text { ein- } \\
\text { schicht. }\end{array}$} & 5.9 & 4.3 & 73 \\
\hline & 33 & 13.5 & & 5.7 & 4.8 & 84 \\
\hline & 24 & 14.6 & & 5.9 & 4.9 & 83 \\
\hline & 21 & 16.4 & & 5.9 & 4.7 & 80 \\
\hline & 18 & 13.8 & & 5.9 & 4.1 & 70 \\
\hline & 15 & 14.7 & & 5.9 & 4.4 & 75 \\
\hline & 15 & 13.1 & & 5.9 & 4.1 & 70 \\
\hline & 15 & 13.7 & & 5.9 & 4.2 & 71 \\
\hline & 12 & 12.9 & & 5.9 & 4.3 & 73 \\
\hline & 12 & 14.5 & & 6.2 & 4.9 & 79 \\
\hline \multirow{10}{*}{$\begin{array}{l}\text { Neu- } \\
\text { geborenes }\end{array}$} & 108 & $\begin{array}{l}. \\
\cdot \quad 8.9\end{array}$ & \multirow{10}{*}{$\begin{array}{l}\text { ein- } \\
\text { schicht. }\end{array}$} & 5.1 & 4.1 & 81 \\
\hline & 99 & 9.5 & & 5.4 & 4.3 & 80 \\
\hline & 93 & 8.9 & & 5.3 & 4.3 & 81 \\
\hline & 78 & 9.4 & & 6.0 & 4.7 & 78 \\
\hline & 72 & 9.6 & & 5.9 & 4.7 & 80 \\
\hline & 57 & 8.8 & & 5.4 & 4.8 & 89 \\
\hline & 39 & 11.8 & & 5.6 & 4.9 & 88 \\
\hline & 36 & 8.4 & & 5.3 & 4.2 & 79 \\
\hline & 33 & 9.2 & & 5.8 & 4.6 & 79 \\
\hline & 30 & 8.0 & & 5.3 & 4.3 & 81 \\
\hline
\end{tabular}


Tabelle 8 (Frotsetzung).

\begin{tabular}{c|r|r|r|r|r|r}
\hline & 231 & 9.6 & & 5.1 & 4.1 & 81 \\
135 & 9.1 & & 5.4 & 4.3 & 78 \\
& 120 & 9.4 & & 5.3 & 4.3 & 81 \\
1 Woche & 87 & 9.1 & & 6.0 & 4.7 & 78 \\
n.P. & 81 & 10.1 & ein- & 5.9 & 4.7 & 80 \\
& 78 & 9.2 & schicht. & 5.4 & 4.8 & 89 \\
& 75 & 8.8 & & 5.6 & 4.9 & 88 \\
& 63 & 9.0 & & 5.3 & 4.2 & 79 \\
48 & 9.4 & & 5.8 & 4.6 & 79 \\
39 & 9.1 & & 5.3 & 4.3 & 81
\end{tabular}

\section{Zusammenfassung.}

Es wurden die Epithelien der Trachea, des Bronchus und der Bronchulen der Menschenembryonen in der zweiten Hälfte der Fetalzeit, der Embryonen von Kaninchen, Meerschweinchen und Ratte in einem letzteren Drittel der Fetalzeit und der Kindchen der genannten Tiere im Alter von 1-3 Wochen histometrisch untersucht, aber vorläufig mit Vorbehalt auf die terminalen Bronchulen mit den Alveolengängen.

1. Über die 'Trachea und den Bronchus. Sie sind in der Fetalzeit bei Mensch mit mehrreihigem Epithel und bei Kaninchen, Meerschweinchen und Ratte mit 2-3 reihigem Epithel ausgekleidet. Bei den Tieren nimmt scine Dicke während der Ent wicklung ab, soweit sie die hier bezogenen Entwicklungsperioden betrifft. Die Dicke des Epithels ordnet sich in den ganzen Entwicklungsperioden in der Reihe: Mensch $>$ Meerschweinchen $>$ Kaninchen $>$ Ratte, wobei der Dickenwert des Meerschweinchens merkwürdigerweise beträchtlich größer ist als derjenige des Kaninchens von weit größerer Körpergröße. Der Wert des Größen-Breitenindexes der im Epithel hoch liegenden Zellkerne ist beim Kaninchen im Bronchus immer etwas größer als in der 'Trachea, beim Meerschweinchen ist aber das Umgekehrte der Fall. In Betreff der Zu- und Abnahme des Indexes während der Entwicklung ist keine allgemeine Regel zu finden.

2. Über die Bronchulen. Das Epithel der Bronchulen, abgesehen von den terminalen, besteht bei Mensch wie bei Kaninchen, Meerschweinchen und Ratte aus einschichtigen Zylinderzellen. Das Bronchulenepithel wird, dasjenige des Meerschweinchens ausgenommen, schon vor dem Partus mehr und mehr dünner. Die peripheren Bronchialäste und die Bronchulen des Meerschweinchens sind in jedem Stadjum gegenüber den anderen Tieren durch ein verhältnismäßig 
dickes Epithel charakterisiert. Der Größen-Breitenindex des Bronchulenepithels zeigt keine regelmäßige Veränderung.

\section{內 容自抄.}

人の胎生後生期，また家鬼，天䇥鼠，大熙鼠の胎生後 3 分 1 期上生後 1-3 週までの莱管, 䒚管支, 絒氣管支の上皮の組織测定を行つた。しかし

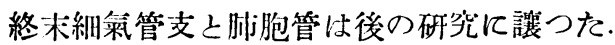

先づ氣管と氣管支につんて云へば，その上.皮は人の胎生期では多列で家 见, 天聯鼠，大熙鼠では $2-3$ 列の上皮である. 動物ではその厚さは發有中

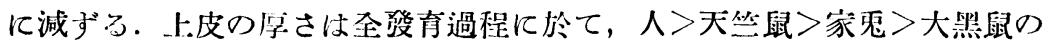
順である.上皮の厚さが芜瓜よりも體形の小さん天些鼠に著しく厚いのが

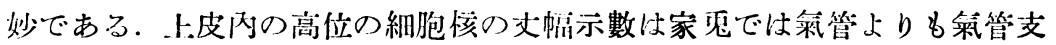
几常にやや大きいが，しかし天然鼠では逆である. 發奇中に於ける丈幅示 數の嵝減には何等一般的な规则が見帅されない。

次にあまり末杪に至らない絒莱管苦について云へば，その上皮は人，芜

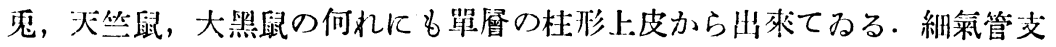
上皮は，天些鼠のるの孝除き，出生前にすでに次第に薄くなる。答鼠の

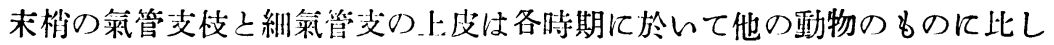
てやや厚い，細氣管支上皮の丈幅示數は規则正しい變化を示さない。

\section{Literatur.}

Bender, K.W.: Über die Entwicklung der Lungen. Z. Anat. 75 (1925). - Ogata, Ch.: Contributions to the histology of the respiratory spaces of the vertebrate lungs. Amer. J. of Anat. 27 (1920). - Policard, R. : Les nonvelles idées sur la disposition de la surface respiratoire pulmonare. Presse méd. 37 (1929). - Shimai, K.: Histogenetische Studien über die Lunge des Menschenembryos. II. (Jap.) Keio I. Z. 28 (1951). 